\title{
A Micro-Macro Traffic Model based on Mean-Field Games
}

\author{
Geoffroy Chevalier, Jerome Le $\mathrm{Ny}^{*}$ and Roland Malhamé
}

\begin{abstract}
Studies of traffic dynamics rely either on macroscopic models considering the traffic as a fluid, or on microscopic models of drivers' behavior. The connection between the microscopic and macroscopic scales is often done via empirical relationships such as the fundamental diagram for macroscopic models, relating traffic flow or average velocity and traffic density. In this paper, we consider a microscopic model consisting of a large number of rational, utility-maximizing drivers interacting on a single road. We then use the theory of Mean Field Games (MFG) to deduce a macroscopic model of traffic density emerging from these interactions. We show how to determine a microscopic utility function for the drivers compatible with standard empirical macroscopic fundamental diagrams. In addition to connecting the microscopic and macroscopic models analytically rather than empirically, our approach can offer additional flexibility to model drivers at the macroscopic level, using a Hamilton-Jacobi-Bellman equation coupled with the standard conservation law for the vehicles.
\end{abstract}

\section{INTRODUCTION}

Traffic modeling is a major area of study in transportation engineering, since the accuracy of traffic predictions depends directly on the quality of the models used [1]. Accurate traffic predictions provide an invaluable service to the drivers, allowing them to estimate their time to destination, and can enable feedback in traffic control laws to mitigate congestion.

Macroscopic traffic models [2], [3] involve two fundamental quantities that depend on space and time, namely the density of vehicles and the average velocity of traffic. Hence, these models must be based on at least two equations. One of them is simply the conservation law for the vehicles, inspired by fluid mechanics. The other one depends on the model chosen. In many cases [4]-[6], studies collect empirical data to draw a fundamental diagram expressing velocity at each location and time as a static function of density, see Fig. 1. A fundamental diagram is typically valid for a given road segment and possibly a time period of the day. Once this fundamental relation is assumed, one can solve the resulting partial differential equation (PDE) to compute the traffic density [1]. Let us also mention second-order models [7], which add a PDE modeling the traffic acceleration instead of postulating a static fundamental diagram.

In addition to macroscopic models, traffic dynamics can also be represented by microscopic models capturing the dynamics of individual cars and their interactions. The most

\footnotetext{
${ }^{*}$ Corresponding author. This work was done while the first author was visiting Polytechnique Montreal.

G. Chevalier is with the Department of Applied Mathematics and Computer Science, ENPC, 77455 Champs-sur-Marne, France. geoffroy.chevalierapolytechnique.org

J. Le Ny and R. Malhamé are with the Department of Electrical Engineering, Polytechnique Montreal, and with GERAD, Montreal, QC H3T 1J4, Canada. jerome.le-ny, roland.malhame@polymtl.ca
}

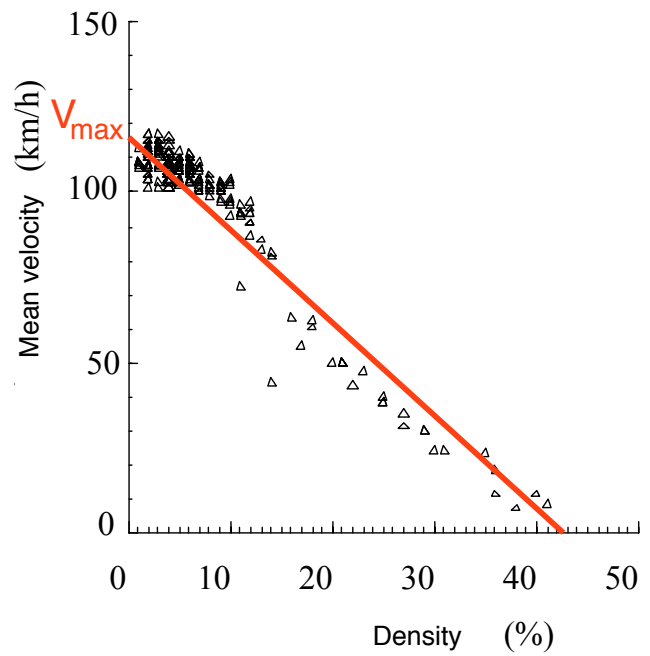

Fig. 1. An example of fundamental diagram, modeling in this case the mean traffic velocity at position $x$ as an affine function of the density of vehicles at $x$. The relationship is typically obtained by least-squares fitting of empirical observations at different locations and times on a given road segment.

popular of these models are leader-follower models [8], where a second-order ordinary differential equation models the dynamics of each car, with the acceleration depending on the separation distance with the car immediately in front of it on the road. Given that the dimension of the state space of the resulting system of differential equations scales linearly with the number of cars, in practice such models are more useful for simulation than mathematical analysis. However, microscopic models have the desirable feature of starting from first principles characterizing individual drivers, hence they allow a priori the modeling of a richer set of behaviors than macroscopic models. Connecting microscopic and macroscopic models is thus of particular interest, as it allows for example to calibrate certain parameters of macroscopic models from observable microscopic quantities. One can show in particular that certain macroscopic models can be obtained as limits in a well-defined sense of leaderfollower models, when the number of vehicles tends to infinity under an appropriate space and time scaling [4], [9].

In this paper we propose a new way of connecting microscopic and macroscopic traffic models, using the approach of Mean-Field Games (MFGs) [10]-[12]. This approach gives us much freedom to define the behavior of individual drivers at a microscopic level as utility-maximizing agents, without restricting ourselves to the structure of leader-follower models. At the same time, MFGs provide a framework to 
derive a corresponding macroscopic model as the number of drivers tends to infinity. In this case, each driver's behavior is influenced by the traffic density through his utility function, and the evolution of the density must be consistent with each driver maximizing his utility. Hence, the evolution of traffic density and velocity is now described by a PDE system composed of a Hamilton-Jacobi-Bellman equation describing the average driver's behavior, in addition to the conservation law. The relationship between velocity and density is thus dynamic as in second-order models. By choosing the mean driver's utility function appropriately, it is possible to fit empirical observations such as the fundamental diagram. This approach allows us to model the drivers in a natural game theoretic framework rather than as simple kinematic particles, which potentially opens the door to translating a rich set of new behaviors to macroscopic models.

The rest of this paper is organized as follows. Section II describes a microscopic game theoretic traffic model with $N$ drivers on a single road segment, which is the standard geometry used to derive classical macroscopic models [1]. In Section III we analyse this problem as $N$ tends to infinity, from the point of view of MFGs. Section IV discusses numerical methods to solve the coupled PDEs of the macroscopic model, examples of simulation results, and an application to fundamental diagram modeling from microscopic parameters. Section V concludes the paper.

\section{Modeling the $N$ Drivers Problem}

We consider a straight road on which $N$ cars indexed by $i \in\{1,2, \ldots, N\}$ are traveling in one direction. We denote by $L$ the length of this road, and consider for simplicity that there is only one lane on the road, so that drivers are not able to overtake each other, as in many standard models [1], [9]. We assume then that the cars are ordered by increasing value of the index $i$, with car $i+1$ traveling in front of car $i$. Driver $i$ 's position is denoted $x_{i}(t) \in \mathbb{R}$, his velocity is $v_{i}(t)$, and his motion extends from $x_{i}(0)=x_{i}^{0}$ to $x_{i}(T)=x_{i}^{f}$, with $T>0$. For simplicity again, the dynamics of each vehicle is described by a single integrator $\frac{d x_{i}(t)}{d t}=v_{i}(t), \quad \forall i \in\{1,2, \ldots, N\}$. The state of the system at time $t$ is given by the collection of car positions $\mathbf{x}(t):=\left[x_{1}(t), \ldots, x_{N}(t)\right]^{T}$, and the vector of drivers' decisions expressing how they set their velocity at time $t$ is given by $\mathbf{v}(t):=\left[v_{1}(t), \ldots, v_{N}(t)\right]^{T}$.

Driver $i$ sets $v_{i}(t)$ so as to maximize a benefit $J_{i, T}^{N}\left(v_{1}, \ldots, v_{N}\right):=\int_{0}^{T} f_{i}^{N}(\mathbf{x}, \mathbf{v}) d t$ defined as the integral over the time horizon $T$ of a utility function denoted $f_{i}^{N}(\mathbf{x}, \mathbf{v}):=f_{i}^{N}\left(x_{1}, \ldots, x_{N}, v_{1}, \ldots v_{N}\right)$. Since this scenario describes a non-cooperative dynamic game, we are interested here in Nash equilibria, where each agent provides a best response given the state and strategy of all other agents. Exact computation of these equilibria for $N$ large is in general a difficult problem.

For concreteness and illustration purposes, in this paper we assume the following utility function for the $i^{t h}$ driver

$$
\begin{aligned}
& f_{i}^{N}(\mathbf{x}, \mathbf{v})=f_{i}^{N}\left(\mathbf{x}, v_{i}\right) \\
& =v_{i}\left(1-F\left(\frac{1}{N} \sum_{j>i} w\left(x_{j}-x_{i}\right)\right)\right)-\frac{1}{2} \alpha v_{i}^{2},
\end{aligned}
$$

which is the sum of three terms. The first term $v_{i}$ models the fact that drivers want to arrive to their destination as fast as possible. The last term $-\alpha v_{i}^{2} / 2$ models the drivers' carefulness when their velocity is too high, independently of the traffic conditions. This term could also model the fact that drivers want to limit their gas consumption, which is proportional to the square or the cube of the velocity in classic fluid friction models. Finally, the term $-v_{i} F\left(\left(\sum_{j>i} w\left(x_{j}-\right.\right.\right.$ $\left.x_{i}\right)$ )/N) models the fact that driver $i$ adapts his velocity to the positions of the cars in front of him, depending on their distances. We introduce a weight $w\left(x_{j}-x_{i}\right)$ for each car $j$ located in front of the $i^{\text {th }}$ driver, where $w$ is a bounded non-negative function on $\mathbb{R}_{+}$. This function $w$ is called the anticipation function in the following (see also [7] for a similar terminology), since it captures how drivers adapt their velocity to anticipate the behavior of cars located in front of them. Note that in contrast to standard leader-follower microscopic models, we do not restrict the influence of the preceding cars on driver $i$ to that of the car immediately preceding him. Finally, the function $F$ is positive and increasing, with $F(0)=0$. To illustrate the usefulness of the model (1), we show below that $F$ can be determined to obtain a velocity-density profile consistent with the empirical data of a fundamental diagram such as the one of Fig. 1. Note that the factor $\frac{1}{N}$ before the sum $\sum_{j>i} w\left(x_{j}-x_{i}\right)$ allows us to bound the influence of the drivers as $N$ becomes large.

Remark 1: In the absence of traffic, a driver's optimal velocity is $v_{\max }:=\frac{1}{\alpha}$. In the presence of traffic, the velocity that maximizes a driver's benefit is always lower than $v_{\max }$.

\section{MEAN FiEld GAME ANALYSiS}

In this section, we consider a sequence of $N$-driver problems as defined previously, with $N$ increasing to infinity. This leads us via a heuristic argument to define a limiting problem corresponding to a Mean-Field Game where a driver adjusts his behavior based on the traffic density in front of him.

Let $\mathcal{F}_{t}^{N}(x)=\frac{1}{N} \sum_{i=1}^{N} \mathbf{1}_{\left\{x_{i}(t) \leq x\right\}}$ be the empirical distribution function of the drivers at time $t \geq 0$, in the $N$-driver problem. Then $\frac{1}{N} \sum_{j>i} w\left(x_{j}-x_{i}\right)=\int_{x_{i}}^{\infty} w\left(y-x_{i}\right) d \mathcal{F}_{t}^{N}(y)$, where the right-hand side is the Lebesgue-Stieltjes integral. If there exists a non-decreasing right-continuous fonction $x \mapsto \mathcal{F}_{t}(x)$ such that $\int_{x_{i}}^{\infty} w\left(y-x_{i}\right) d \mathcal{F}_{t}^{N}(y) \rightarrow \int_{x_{i}}^{\infty} w(y-$ $\left.x_{i}\right) d \mathcal{F}_{t}(y)$ as $N$ tends to infinity, and if moreover the measure on $\mathbb{R}$ defined by $\mu_{t}((a, b])=\mathcal{F}_{t}(b)-\mathcal{F}_{t}(a)$ admits a density $m_{t}(x)$ with respect to the Lebesgue measure, then we have

$$
\lim _{N \rightarrow \infty} \frac{1}{N} \sum_{j>i} w\left(x_{j}-x_{i}\right) \rightarrow \int_{x_{i}}^{\infty} w\left(y-x_{i}\right) m_{t}(y) d y .
$$


In this case $m_{t}(x)$ represents the number of cars in the interval $[x, x+d x]$. We make the assumption in this paper that such a density exists.

We are thus led to the following limit problem for each generic agent or "mean player", which we call the MeanField Game (MFG)

$$
\max _{v(\cdot)} J_{T}(v):=\int_{0}^{T} f\left(m_{t}, x(t), v(t)\right) d t
$$

where $\left\{m_{t}, t \in[0, T]\right\}$ is an (a priori unknown) flow of traffic density functions, the mean player dynamics is $\frac{d x}{d t}=v$ and his utility function is

$$
f(m, x, v)=v\left(1-F\left(\int_{x}^{\infty} m(y) w(y-x) d y\right)\right)-\frac{1}{2} \alpha v^{2} .
$$

\section{A. Myopic Drivers}

Before providing a solution for the previous MFG problem, in this subsection we consider the behavior of an average driver who only maximizes its instantaneous utility $f\left(m_{t}, x(t), v(t)\right)$ at each time instead of $J_{T}$, which corresponds to a model of bounded rationality. Simply remarking that $f$ is concave in $v$, the instantaneous myopic velocity is then obtained as

$$
\begin{aligned}
& \frac{\partial f\left(m_{t}, x(t), v\right)}{\partial v}=0 \Longleftrightarrow \\
& v(t)=\frac{1}{\alpha}\left(1-F\left(\int_{x(t)}^{\infty} m_{t}(y) w(y-x(t)) d y\right)\right) .
\end{aligned}
$$

We can use this myopic solution for example to choose $F$ in order to fit the empirical data of Fig. 1 say, i.e.,

$$
v(t)=v_{\max }\left(1-\frac{m_{t}(x(t))}{m_{\mathrm{lim}}}\right), \forall t,
$$

assuming again myopic behavior of the drivers. Here $m_{\mathrm{lim}}$ is the traffic jam density and $v_{\max }$ the maximal traffic velocity. A possible choice for $F$ and $w$ is

$$
w(y)=\delta(y), \quad F(z)=\frac{z}{m_{\lim }},
$$

where $\delta$ denotes the Dirac delta. Note that the fundamental diagram makes the assumption that the average traffic velocity at $x$ does not depend on the traffic conditions in front of $x$, but only on the density at $x$.

The empirically fitted profile of Fig. 1 gives $v(t)$ only as a function of $m_{t}(x(t))$. With our model, we can have such a profile when choosing $w(x)=\delta(x)$. Keeping the same function $F$ as in (4) but using $w$ to take into account the upstream traffic, we obtain the following more general relationship between velocity and density

$$
v(t)=v_{\max }\left(1-\int_{x(t)}^{\infty} \frac{m_{t}(y)}{m_{l i m}} w(y-x(t)) d y\right) .
$$

\section{B. Nash-MFG Equilibrium}

We now return to the MFG problem and the maximization of the utility function by each individual over the entire time horizon. Since we are facing a game, the solution concept considered needs to be defined. The Nash-MFG equilibrium is defined as the situation in which the "mean driver" sets its velocity to maximize his integral utility $J_{T}$ while anticipating the behavior of the mean field, that is to say, the evolution of the density of the other drivers. Since each driver is doing the same, to have an equilibrium the dynamics of this density must be consistent with the control actions of the individuals. We define for $t \in[0, T]$ the value function

$$
V_{t}(x)=\max _{v(\cdot)} \int_{t}^{T} f\left(m_{\tau}, x(\tau), v(\tau)\right) d \tau
$$

For the existence and unicity of a Nash-MFG equilibrium, we must make some additional assumptions.

Assumption 1: $F$ is Lipschitz on its domain of definition, and $m_{0}$ is absolutely continuous and has a second order moment, i.e., $\int_{\mathbb{R}} y^{2} m_{0}(y) d y<+\infty$.

Theorem 1: Under Assumption 1, the following PDE system

$$
\begin{aligned}
& \frac{\partial V_{t}(x)}{\partial t}+\max _{v}\left\{f\left(m_{t}, x, v\right)+v \frac{\partial V_{t}(x)}{\partial x}\right\}=0 \\
& \frac{\partial m_{t}(x)}{\partial t}+\frac{\partial\left(m_{t}(x) v_{t}(x)\right)}{\partial x}=0 \\
& V_{T}(x)=0, m_{0}(x) \text { given, for all } x,
\end{aligned}
$$

where $v_{t}(x)$ in (CL) is the maximizing value of $v$ in (HJB), has a unique solution $(x, t) \mapsto\left(V_{t}(x), m_{t}(x)\right)$. We call this solution together with $(x, t) \mapsto v_{t}(x)$ the Nash-MFG equilibrium.

The proof of Theorem 1 follows [13, Theorem 3.1]. Equation (HJB) is a Hamilton-Jacobi-Bellman equation and (CL) is a conservation law for the drivers' density. Note also that for the assumed utility fonction (2), which is quadratic in $v$, the maximization in (HJB) can be performed explicitly to yield

$v_{t}(x)=\frac{1}{\alpha}\left(1-F\left(\int_{x}^{\infty} m_{t}(y) w(y-x) d y\right)+\frac{\partial V_{t}(x)}{\partial x}\right)$,

and thus the $\max _{v}$ can be removed from the equation by substitution of this solution.

Remark 2: The quantity $v(t)$ in the previous sections is a Lagrangian velocity, i.e., the velocity of a moving driver with trajectory $x(t)$. Theorem 1 provides an Eulerian velocity field $v_{t}(x)$, i.e., the mean-driver's velocity at location $x$ at time $t$. The relation between the two quantities is $v(t)=v_{t}(x(t))$.

\section{Simulations AND RESUlts}

In this section, we discuss numerical schemes to compute a Nash-MFG equilibrium, as well as some simulation results. Since it is more convenient to work with dimensionless variables, we define $\rho_{t}(x)=\frac{m_{t}(x)}{m_{\text {lim }}}$, where $m_{\text {lim }}$ was defined above (4). We take (with a slight abuse of notation) $F(\rho)=\rho$ to be the function (4), found to be compatible with an affine 
velocity/density relationship under the myopic solution, and redefine

$$
f(\rho, x, v)=v\left(1-\int_{x}^{\infty} \rho(y) w(y-x) d y\right)-\frac{1}{2} \alpha v^{2}
$$

accordingly. The triple $\left\{\rho_{t}(x), v_{t}(x), V_{t}(x)\right\}$ is the solution of the PDE system

$$
\begin{aligned}
& \frac{\partial V_{t}(x)}{\partial t}+\max _{v}\left\{f\left(\rho_{t}, x, v\right)+v \frac{\partial V_{t}(x)}{\partial x}\right\}=0 \\
& \frac{\partial \rho_{t}}{\partial t}(x)+\frac{\partial\left(\rho_{t}(x) v_{t}(x)\right)}{\partial x}=0 \\
& V_{T}(x)=0, \quad \rho_{0}(x) \text { given }
\end{aligned}
$$

where $v_{t}(x)$ is the result of the maximization in (7a), which is computable explicitly as explained in the previous section.

\section{A. Backward-forward iterations}

System (7) is a backward-forward nonlinear PDE system. To solve it numerically, we solve (7a) backwards, starting from $V_{T}(x)=0$, assuming initially $\rho_{t}(x)=\rho_{0}(x)$, for all $t \in[0, T]$. We then inject the solution $v^{(1)}$ found after this step in the forward conservation equation (7b) to find a new solution $\rho_{t}(x)$ denoted $\rho^{(1)}$, assuming the initial condition $\rho_{0}(x)$ and $v=v^{(1)}$. We continue to iterate these two steps until the solutions converge to a fixed point.

To solve numerically this system, we introduce the stepsizes $\Delta x=\frac{L}{m}$ and $\Delta t=\frac{T}{n}$ where $m, n$ are integers. We define $\rho_{j}^{i}, v_{j}^{i}$ and $V_{j}^{i}$ to be the approximations of $\rho_{j \Delta t}(i \Delta x)$, $v_{j \Delta t}(i \Delta x)$ and $V_{j \Delta t}(i \Delta x)$ for $i \in\{0, \ldots, m\}$ and $j \in$ $\{0, \ldots, n\}$. The following finite differences scheme is used to solve the backward HJB equation [14]

$$
\left\{\begin{array}{l}
\frac{V_{j+1}^{i}-V_{j}^{i}}{\Delta t}+\frac{1}{2} \alpha\left(v_{j}^{i}\right)^{2}=0 \\
v_{j}^{i}=\frac{1}{\alpha}\left(1-\tilde{\rho}_{j}^{i}+\frac{V_{j}^{i+1}-V_{j}^{i}}{\Delta x}\right)
\end{array}\right.
$$

where $\tilde{\rho}_{j}^{i}=\sum_{k \geq i} \rho_{j}^{k} w((k-i) \Delta x) \Delta x$ is the numerical approximation for $\int_{i \Delta x}^{\infty} \rho_{j \Delta t}(y-i \Delta x) w(y) d y$.

We solve the conservative equation (7b) with a finite volume conservative Lax-Wendroff scheme [15]. Define

$$
\rho_{j}^{i}=\frac{1}{\Delta x} \int_{x_{i-1 / 2}}^{x_{i+1 / 2}} \rho_{j \Delta t}(x) d x
$$

where $x_{i \pm 1 / 2}=\left(i \pm \frac{1}{2}\right) \Delta x$. Defining $\rho^{i}(t)=$ $\frac{1}{\Delta x} \int_{x_{i-1 / 2}}^{x_{i+1 / 2}} \rho_{t}(x) d x$ and integrating (7b) between $x_{i-1 / 2}$ and $x_{i+1 / 2}$, we obtain

$$
\begin{aligned}
\frac{d \rho^{i}}{d t}(t)= & -\frac{1}{\Delta x}\left\{\rho_{t}\left(x_{i+1 / 2}\right) v\left(x_{i+1 / 2}, t\right)\right. \\
& \left.-\rho_{t}\left(x_{i-1 / 2}\right) v\left(x_{i-1 / 2}, t\right)\right\} .
\end{aligned}
$$

Integrating this equation between $j \Delta t$ and $(j+1) \Delta t$, we get

$$
\begin{aligned}
\rho_{j+1}^{i}= & \rho_{j}^{i}-\frac{1}{\Delta x} \int_{j \Delta t}^{(j+1) \Delta t}\left\{\rho_{t}\left(x_{i+1 / 2}\right) v\left(x_{i+1 / 2}, t\right)\right. \\
& \left.-\rho_{t}\left(x_{i-1 / 2}\right) v\left(x_{i-1 / 2}, t\right)\right\} d t .
\end{aligned}
$$

The Lax-Wendroff method consists in doing the following approximation

$$
\begin{aligned}
\int_{j \Delta t}^{(j+1) \Delta t} \rho_{t}\left(x_{i+1 / 2}\right) v & \left(x_{i+1 / 2}, t\right) d t \approx \frac{\Delta t}{2}\left(\rho_{j}^{i} v_{j}^{i}+\rho_{j}^{i+1} v_{j}^{i+1}\right) \\
& -\frac{(\Delta t)^{3}}{2 \Delta x} \frac{v_{j}^{i}+v_{j}^{i+1}}{2}\left(\rho_{j}^{i+1} v_{j}^{i+1}-\rho_{j}^{i} v_{j}^{i}\right) .
\end{aligned}
$$

We then get the following explicit scheme for the conservation equation (7b)

$$
\begin{array}{r}
\rho_{j+1}^{i}=\rho_{j}^{i}-\frac{\Delta t}{2 \Delta x}\left(\rho_{j}^{i+1} v_{j}^{i+1}-\rho_{j}^{i-1} v_{j}^{i-1}\right) \\
+\frac{1}{2}\left(\frac{\Delta t}{\Delta x}\right)^{2}\left\{\frac{v_{j}^{i}+v_{j}^{i+1}}{2}\left(\rho_{j}^{i+1} v_{j}^{i+1}-\rho_{j}^{i} v_{j}^{i}\right)\right. \\
\left.-\frac{v_{j}^{i-1}+v_{j}^{i}}{2}\left(\rho_{j}^{i} v_{j}^{i}-\rho_{j}^{i-1} v_{j}^{i-1}\right)\right\} .
\end{array}
$$

For the scheme to be consistent (in this case, to the second order), we must also impose the Courant-Friedrichs-Lewy condition on the stepsizes, i.e., $\Delta t<\alpha \Delta x$ [15].

\section{B. Convergence of the algorithm}

Let $\rho^{(k)}=\left\{\left(\rho_{j}^{i}\right)_{k}, i \in\{0, \ldots, m\}, j \in\{0, \ldots, n\}\right\}$ be the solution obtained after $k \in \mathbb{N}$ backward-forward iterations. To show numerically that the algorithm converges to a fixed function, we consider the squared $L^{2}$ distance between $\rho^{(k+1)}$ and $\rho^{(k)}$

$\epsilon_{k}^{2}=\left\|\rho^{(k+1)}-\rho^{(k)}\right\|^{2}=\sum_{j=0}^{n} \sum_{i=0}^{m}\left(\left(\rho_{j}^{i}\right)_{k+1}-\left(\rho_{j}^{i}\right)_{k}\right)^{2} \Delta x \Delta t$.

If this quantity converges to zero fast enough, the algorithm converges to a fixed function.

Proposition 1: The forward-backward algorithm converges if $\sum_{k \geq 0} \epsilon_{k}<\infty$.

Proof: By the triangle inequality, we have for $k, p \in \mathbb{N}$

$$
\begin{aligned}
\left\|\rho^{(k+p)}-\rho^{(k)}\right\| & \leq \sum_{l=0}^{p-1}\left\|\rho^{(k+l+1)}-\rho^{(k+l)}\right\|_{L^{2}} \\
& \leq \sum_{l=0}^{p-1} \epsilon_{k+l} \leq \sum_{l \geq k} \epsilon_{l} .
\end{aligned}
$$

The last quantity tends to 0 as $k$ tends to infinity. Therefore, $\left\{\rho^{(k)}\right\}_{k \in \mathbb{N}}$ converges since it is a Cauchy sequence.

Corollary 1: If there is $a>0$ and $b \in \mathbb{R}$ such that $\log \epsilon_{k}<b-a k$, the forward-backward algorithm converges.

\section{Numerical results}

1) Dirac anticipation function: We first compute solutions for an initially non-saturated traffic, with $\rho_{0}$ having a (truncated) Gaussian profile, $\max _{x} \rho_{0}(x)=\frac{1}{4}$, and a Dirac anticipation function $w=\delta$. Fig. 2 gives an example of traffic evolution for the Nash-MFG equilibrium. We see that the maximal density decreases over time, which means that traffic tends to get less congested, as expected. Fig. 3 shows the final velocity distribution at $t=T$ as a function of $x$, confirming that the velocity decreases with higher density. 


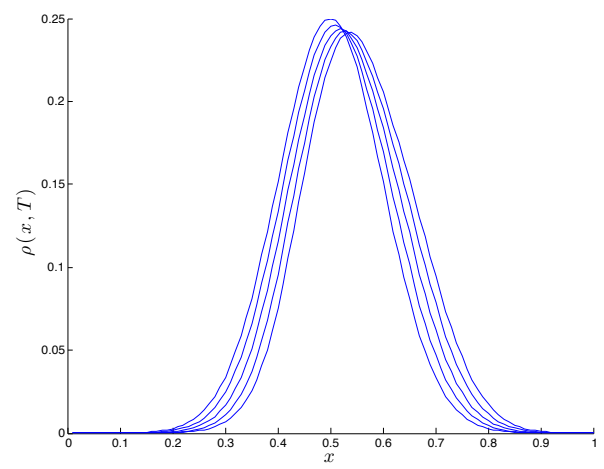

Fig. 2. Evolution of the traffic density at different times $(t=0, t=T / 4$, $t=T / 2, t=3 T / 4$ and $t=T$ ) with initially non-saturated traffic and a Dirac anticipation function. This result was computed with the following parameters $T=1, v_{\max }=0.07, \rho_{0}(x)=\frac{1}{4} e^{-\left(\frac{x-\mu}{\sigma}\right)^{2}}$, with $\mu=0.5$ and $\sigma=0.1$ and $n=100, w(x)=\delta(x), F(\stackrel{\rho}{\rho})=\rho$, and 20 iterations.

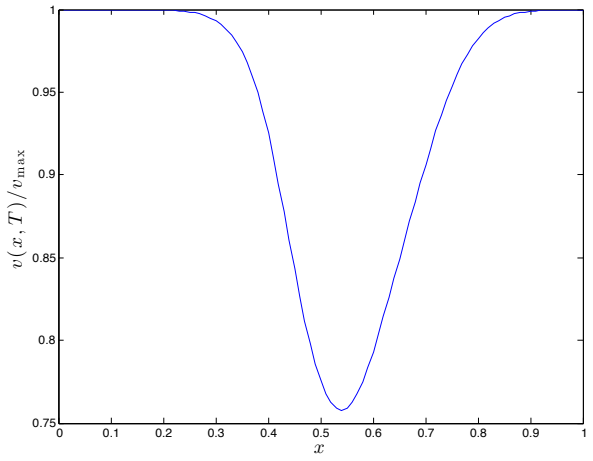

Fig. 3. Profile of the final velocity $v_{T}(x)$, for the scenario of Fig. 2.

The fact that the distribution is symmetric with respect to its maximum point is expected, since with $w=\delta$ the drivers do not account for the density in front of them to set their velocity. The convergence of the algorithm is illustrated on Fig. 4. We see that $\log \epsilon_{k}$ seems to be decreasing linearly asymptotically, which means from Corollary 1 that the algorithm converges to a fixed point.

2) Exponentially decaying anticipation function: We repeat the preceding simulations, but for a more realistic anticipation function, in this case

$$
w(y)=\frac{1}{\lambda} e^{-\frac{y}{\lambda}}
$$

with $\lambda$ a positive parameter. We choose $\lambda$ somewhat arbitrarily for illustration purposes to be $0.1 \sqrt{\operatorname{Var}\left(X_{0}\right)}$ where $\operatorname{Var}\left(X_{0}\right)=\int_{\mathbb{R}}\left(x-\mathbb{E}\left(X_{0}\right)\right)^{2} \rho_{0}(x) d x$, with $\mathbb{E}\left(X_{0}\right)=$ $\int_{\mathbb{R}} x \rho_{0}(x) d x$. The results of this simulation are presented on Fig. 5 and 6. We see in particular that the velocity profile becomes non-symmetric, because of the drivers now adjusting their velocity to the traffic density in front of them, in particular slowing down sooner as they sense the higher density upstream.

3) Comparison between the anticipation functions and fundamental diagram: Fig. 7 attempts to illustrate the relationship between velocity and density similarly to the fundamental diagram, when $w$ is a Dirac (blue curve) and when

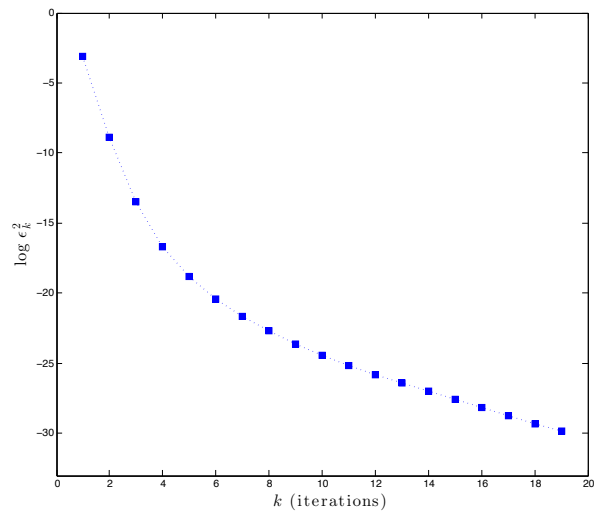

Fig. 4. Evolution of the quantity $\log \epsilon_{k}^{2}$ as a function of the number of iterations $k$. The condition of the corollary 1 appears to be met.

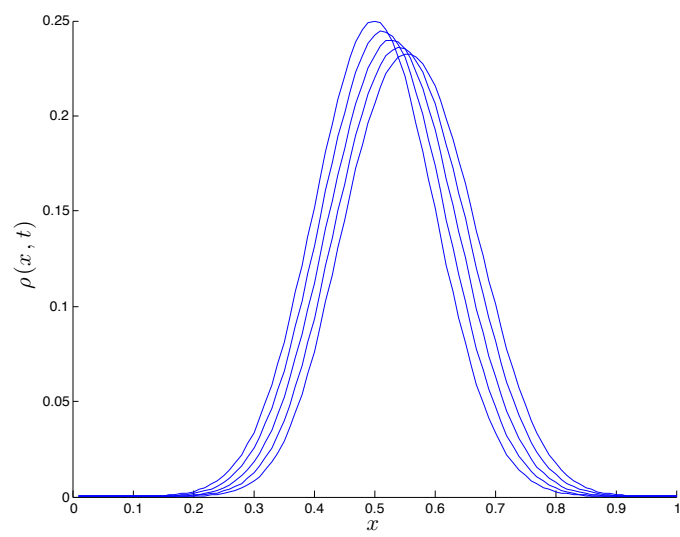

Fig. 5. Evolution of the traffic density from $t=0$ to $t=T$ for the same parameters as on Fig. 2, except for $w(y)=\frac{1}{\lambda} e^{-\frac{y}{\lambda}}$.

$w$ is the exponentially decaying function (8) (red curves). In the second case, we represented two curves corresponding to the situations where the driver is behind or in front of the density spike. Drivers located in front of the spike, who do not see a higher traffic density in front of them, drive faster when their anticipation function is not a simple Dirac function, which is intuitively plausible. Mathematically, this is explained by the fact that $\int_{x}^{\infty} \rho_{t}(y) w(y-x) d y<\rho_{t}(x)$ when $\rho_{t}(\cdot)$ is decreasing in $x$, since $\int_{0}^{\infty} w(y) d y=1$. By the same reasoning, we obtain that drivers located behind the density spike drive more slowly as they anticipate the slower upcoming traffic. This phenomenon caused by the anticipation function provides thus one explanation for the dispersion seen in Fig. 1 of the density-velocity pairs around the fundamental diagram.

Our final result shows that for small values of $\lambda$, the difference between the red and the blue curves caused by the anticipation function on Fig. 7 is approximatively given by $v_{\max } \lambda \frac{\partial \rho_{t}(x)}{\partial x}$. This means that drivers will adapt their velocity proportionally to $\lambda$. Although this result is shown for the myopic velocity (3), which differs from the optimal solution (6) by the term $\frac{\partial V_{t}(x)}{\partial x}$, in this simulation the latter term turns out to be small and the two solutions are close. This can be seen in the case of $w=\delta$ since the blue curve 


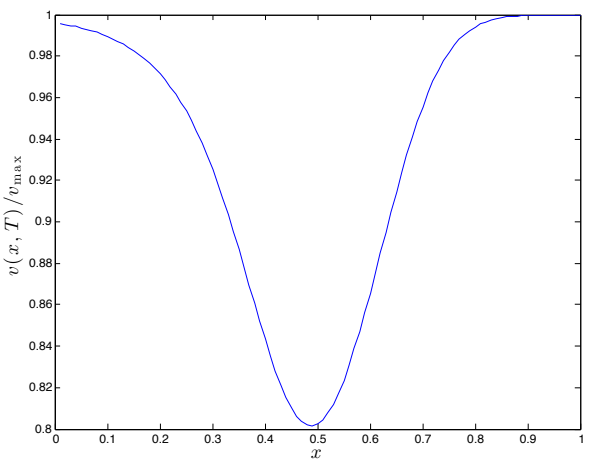

Fig. 6. Profile of the final velocity $v(x, t=T)$ for the scenario of Fig. 5 .

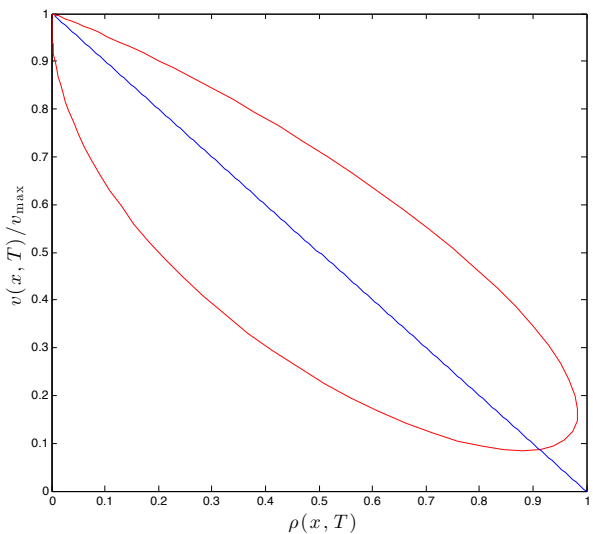

Fig. 7. Fundamental diagram representing the velocity as a function of the density at the same point. The straight line is obtained with $w=\delta$ and the double curve, with $w(y)=\frac{1}{\lambda} e^{-\frac{y}{\lambda}}$ and $\lambda=0.1 \sqrt{\operatorname{Var}\left(\rho_{0}\right)}$. Both curves were computed with the same parameters otherwise.

is very close to a line.

Proposition 2: Suppose that $\rho_{t}(\cdot)$ is $n$ times continuously differentiable and $w$ is given by (8). Suppose that for each $x \in \mathbb{R}$ and for each $t \in[0, T],\left|\frac{\partial^{n} \rho_{t}(x)}{\partial x^{n}}\right|<M$. Finally, suppose that the drivers are myopic, setting their velocity to $v_{t}(x)=v_{\max }\left(1-\int_{x}^{\infty} \rho_{t}(y) w(y-x) d y\right)$, as in (5). Then we have $v_{t}(x)=v_{\max }\left(1-\rho_{t}(x)-\sum_{k=1}^{n-1} \lambda^{k} \frac{\partial^{k} \rho_{t}(x)}{\partial x^{k}}\right)+$ $O\left(\lambda^{n}\right)$.

Proof: Using Taylor-Lagrange formula, for $x \in \mathbb{R}$ and $y>x$, there is $\xi \in] x, y[$ such that

$\rho_{t}(y)=\rho_{t}(x)+\sum_{k=1}^{n-1} \frac{(y-x)^{k}}{k !} \frac{\partial^{k} \rho_{t}(x)}{\partial x^{k}}+\frac{(y-x)^{n}}{n !} \frac{\partial^{n} \rho_{t}(\xi)}{\partial x^{n}}$.

By the definition of the $\Gamma$ function, we have $\int_{0}^{\infty} y^{k} e^{-y} d y=$ $k$ !, and hence

$$
\begin{aligned}
& v_{t}(x)=v_{\max }\left(1-\int_{x}^{\infty} \rho_{t}(y) w(y-x) d y\right) \\
& =v_{\max }\left(1-\sum_{k=0}^{n-1} \frac{\partial^{k} \rho_{t}(x)}{\partial x^{k}} \int_{x}^{\infty} \frac{(y-x)^{k}}{k !} e^{-\frac{(y-x)}{\lambda}} d y\right) \\
& +v_{\max } \int_{x}^{\infty} \frac{(y-x)^{n}}{n !} \frac{\partial^{n} \rho_{t}(\xi)}{\partial x^{n}} e^{-\frac{y-x}{\lambda}} d y
\end{aligned}
$$

$$
\begin{aligned}
v_{t}(x) & =v_{\max }\left(1-\rho_{t}(x)-\sum_{k=1}^{n-1} \lambda^{k} \frac{\partial^{k} \rho_{t}(x)}{\partial x^{k}}\right) \\
& +v_{\max } \int_{x}^{\infty} \frac{(y-x)^{n}}{n !} \frac{\partial^{n} \rho_{t}(\xi)}{\partial x^{n}} e^{-\frac{y-x}{\lambda}} d y .
\end{aligned}
$$

Finally, by triangle inequality :

$$
\left|\int_{x}^{\infty} \frac{(y-x)^{n}}{n !} \frac{\partial^{n} \rho_{t}(\xi)}{\partial x^{n}} e^{-\frac{y-x}{\lambda}} d y\right| \leq M \lambda^{n} .
$$

\section{CONCLUSIONS}

In this paper, we formulated an $N$-driver game-theoretic problem to model the traffic dynamics on a single road. In this model, fully rational drivers aim at maximizing the integral of their utility function. We then defined a corresponding Mean-Field Game, justified intuitively as the limit of the $N$-driver model when $N$ goes to infinity. This new "secondorder" traffic model adds to the conservation law a backward HJB equation representing the drivers' behavior. We also showed that myopic drivers in this framework are sufficient to explain the fundamental diagram of a first order traffic model, by setting the drivers' utility function appropriately. Future work will investigate more formally the link between the MFG model and the model with $N$ finite, in particular the concept of $\epsilon$-Nash equilibrium for the latter [11].

\section{REFERENCES}

[1] M. Treiber and A. Kesting, Traffic Flow Dynamics. Springer, 2013.

[2] M. Lighthill and G. Whitham, "On kinematic waves II. A theory of traffic flow on long crowded roads," Proceedings of the Royal Society, vol. 229A, pp. 317-345, May 1955.

[3] P. I. Richards, "Shockwaves on the highway," Operations Research, vol. 4, pp. 42-51, 1956.

[4] S. J. Al Nasur, "New models for crowd dynamics and control," Ph.D. dissertation, Virginia Polytechnic Institute and State University, December 2006.

[5] 75 years of the Fundamental Diagram for Traffic Flow Theory (Greenshields Symposium). Transportation Research Board, 2011.

[6] N. Bellomo and C. Dogbe, "On the modeling of traffic and crowds: a survey of models, speculations and perspectives," SIAM Review, vol. 53, pp. 409-463, August 2011.

[7] A. Aw and M. Rascle, "Resurrection of "second order" models of traffic flow," SIAM Journal on Applied Mathematics, vol. 60, no. 3, pp. 916-938, 2000.

[8] D. Gazis, R. Herman, and R. Rothery, "Nonlinear follow-the-leader models of traffic flow," Operations Research, vol. 9, no. 4, pp. 545567, July-August 1961.

[9] A. Aw, A. Klar, T. Materne, and M. Rascle, "Derivation of continuum traffic flow models from microscopic follow-the-leader models," SIAM Journal on Applied Mathematics, vol. 63, no. 1, pp. 259-278, 2002.

[10] M. Huang, P. E. Caines, and R. Malhamé, "Individual and mass behaviour in large population stochastic wireless power control problems: centralized and nash equilibrium solutions," in Proceedings of the 42nd IEEE Conference on Decision and Control, December 2003.

[11] M. Huang, P. E. Caines, and R. P. Malhamé, "Large-population cost-coupled LQG problems with nonuniform agents: individual-mass behavior and decentralized $\epsilon$-nash equilibria," IEEE Transactions on Automatic Control, vol. 52, no. 9, pp. 1560-1571, September 2007.

[12] J. M. Lasry and P. L. Lions, "Mean field games," Japanese Journal of Mathematics, vol. 2, pp. 229-260, 2007.

[13] P. Cardaliaguet, "Notes on mean field games," September 2013, lecture notes from P.-L. Lions' lectures at Collège de France.

[14] Y. Achdou and I. Capuzzo-Dolcetta, "Mean field games: Numerical methods," SIAM Review, vol. 48, pp. 1136-1162, 2010.

[15] R. J. LeVeque, Numerical Methods for Conservation Laws, 2nd ed. Birkhäuser, 1992. 